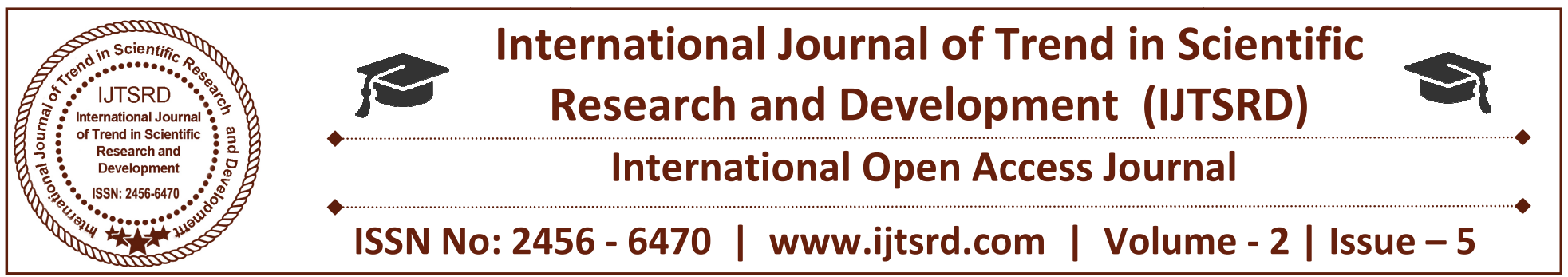

\title{
Antenna Positioning Based on IOT
}

\author{
Khalid Makhdoomi \\ Assistant Professor \\ Department of Computer Science and Engineering, \\ SSM College of Engineering and Technology, Baramulla, Jammu and Kashmir, India
}

\begin{abstract}
Information Technology change the world we live in like connected cars, industries with smart machinery, e-commerce, banking etc. the IOT and Microcontrollers are the most popular technological concepts and have a great importance in every domain of life. The paper describes how to deploy latest and emerging technologies in Antenna Positioning.
\end{abstract}

\section{KEYWORD: Sensor, IOT}

\section{INTRODUCTION}

IOT is one of the latest and emerging technology with the ability to transform the beautiful world like industries with smart machines, smarter cities etc. However, the importance of technology like IOT in communication systems has very great impact. In wireless communication concept antennas are very important for receiving signals. For very effective results of wireless communication the proper direction of antennas matters. So in my research I propose a very dynamic model for antenna positioning based on IOT technology. Sensors will be mounted on the antenna to detect the proper direction and the interesting thing is the motors using IOT concept will change the direction from anywhere across the world. The direction of transmitting stations changes with time means when the transmitting station changes over time the antenna direction need to be automatically changes accordingly. In simple words this model will help us in monitoring the direction of antennas and transmitting new coordinates to properly position the antennas. The internet of things (IoT) being a fascinating and exciting concept has one of the major challenging aspects of having a secure ecosystem encompassing all building blocks of IoT architecture. RFID is a promising technology for the proliferation of IoT, and it can be used to detect and identify the items. In IoT (Internet of things), "things" refer to a wide range of devices such as heart monitoring implants, remotely handling home appliances, biochip transponders used on farm animals, cameras that are streaming live feeds of wild animals in coastal waters etc. Thus we can say that "things" are a "mixture of hardware, software, data and services". System engineering of a satellite based data communication baseline concept is presented to achieve terabit per second throughput. Along with IoT

if sensors and actuators are augmented, the technology becomes as more general class of cyberphysical systems which can also encompass the technologies such as smart grids, virtual power plants, smart homes, intelligent transportation and smart cities. As we know wireless communication systems work on antennas for reception of signals. It is necessary to properly position the antennas in the direction of the transmitters for effective communication.

\section{Problem Formulation}

The present world is full with wireless communication systems and number of technologies is used to make the wireless communication system more and more dynamic. IOT is also one of the leading and fast growing technologies. For proper wireless communication antennas are considered very important devices that receive signals for transmission. The signal received by antennas is affected by the direction of transmitting stations those changes over time so there is a need of system that automatically changes the antenna positioning if there is any change in transmitting station over time. 


\section{Objectives}

The main objectives of my research are described below:

1. To implement microcontroller based concept in wireless communication.

2. To make reduce of the poor signaling.

3. To access the system from anywhere across the globe.

\section{Proposed Methodology}

All wireless systems for communications use antennas for signal receiving and for effective performance of wireless communication proper positioning is very important. So in this paper I am describing a model based on IOT for automatic antenna positioning. In my model we use sensors and mount motors on each antenna to verify its facing direction transmitted over IOT. In case the direction of satellite or station changes with time the direction of antenna also changes accordingly. The antenna positions are visible to operator on the IOT GUI through the internet. The IOT Gecko is used to develop the GUI system for antenna monitoring. Our model allows for monitoring antenna direction as well as transmitting new coordinates to position the antenna and motor appropriately positions the antenna accordingly.

\section{Proposed Block Diagram}

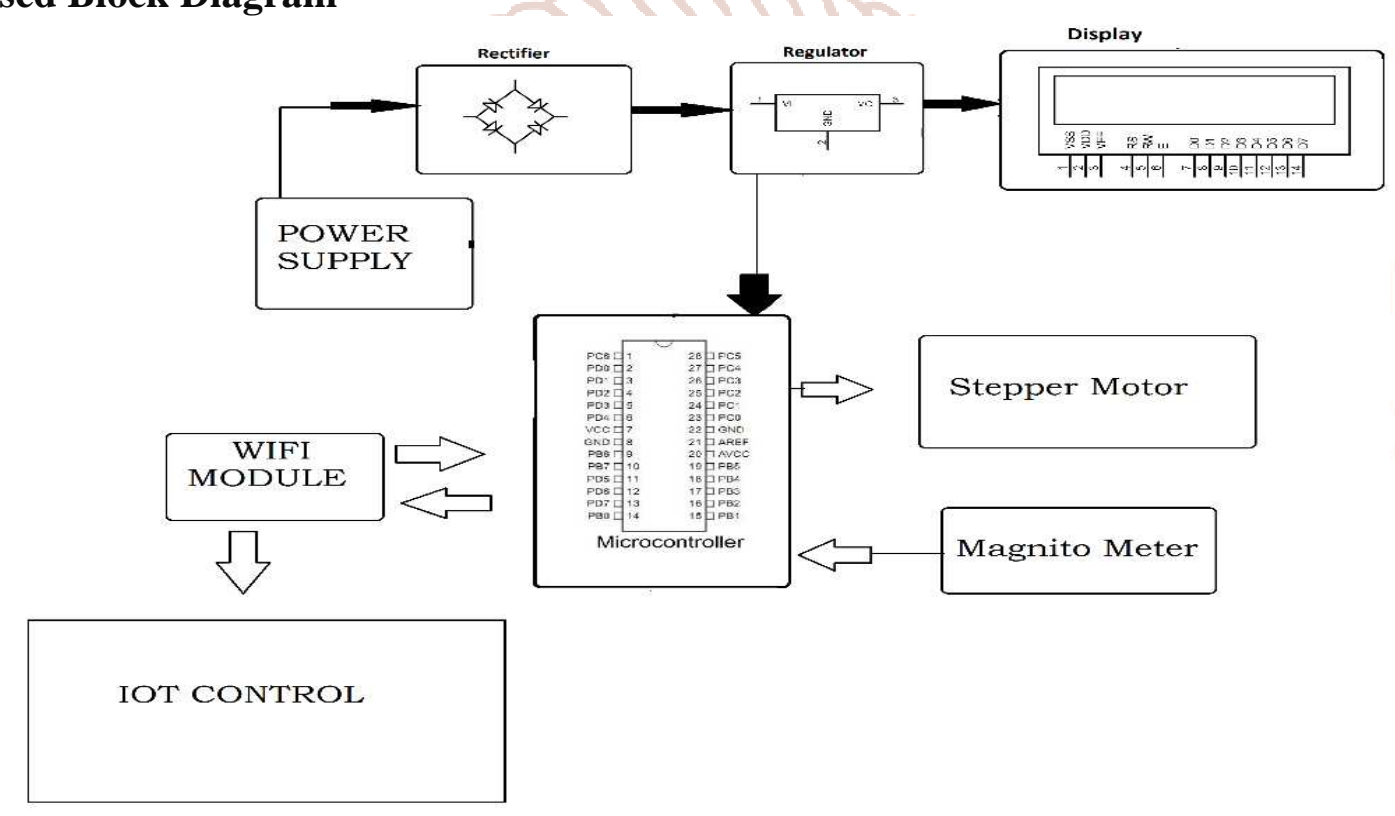

\section{CONCLUSION}

The IOT is a latest and emerging technology with great significance in every domain of life. The paper describes the implementation of latest technologies in antenna positioning. The IOT Gecko is used to develop the GUI system for antenna monitoring. This model allows for monitoring antenna direction as well as transmitting new coordinates to position the antenna and motor appropriately positions the antenna accordingly.

\section{REFERENCES}

1. R. L Sturdivant, "Systems Engineering of a Terabit Elliptic Orbit satellite", published in IEEE, volume 4, pp 9941-9949 Sep 2016.

2. BekirSaitCiftler \& Abdullah Kadri, "IoT Localization for Bistatic Passive UHF RFID Systems With 3-D Radiation Pattern", published in IEEE, Volno4, pp 905-913, August 2017.
3. S. Katoch, H. Jotwani, S. Pani, A. Rajawat, "A Compact Dual Band Antenna for IoT Applications", published in IEE, pp1594-1597, 2015.

4. Alan Bensky, "Wireless positioning and applications (Third edition)", British library Publications.

5. McHugh J. M and Konrad J. and Saligrama V and Jodoin P, Foreground-Adaptive Background Subtraction, IEEE Signal Processing Letters, 16, Issue 5, May-2009,390-393

6. Dnyanada Jadhav and Prof. L. M. R. J. Lobo, Hand Gesture Recognition System to Control Slide Show Navigation, International Journal of Application or Innovation in Engineering and Management, Issue 1, Jan 2014, 283-286 
7. Siddharth S. Rautaray and Anupam Agrawal, Interaction with Virtual Game through Hand Gesture Recognition, International Conference on Multimedia, Signal Processing and Communication Technologies, 11, 2011, 244-247

8. C. H. Bennett, Logical of computation, IBM Journal of Research and Development, 117, 63, Nov-1993, 5205- 5302

9. P. N. V. S. Gowtham, A Hand Gesture Recognition Based Virtual Touch Wall, International Journal of Information and Education Technology, 2, Feb-2012, 36-42
10. Juan Wachs and Helman Stern and Yael Edan and Michael Gillam and Craig Feied and Mark Smith, A Real Time Hand Gesture Interface for Medical Visualization Applications, Jon Handler2 Applications of Soft Computing, 36, 153-162

11. Feng-Sheng Chen, Chih-Ming Fu, Chung-Lin Huang, Hand gesture recognition using a real time

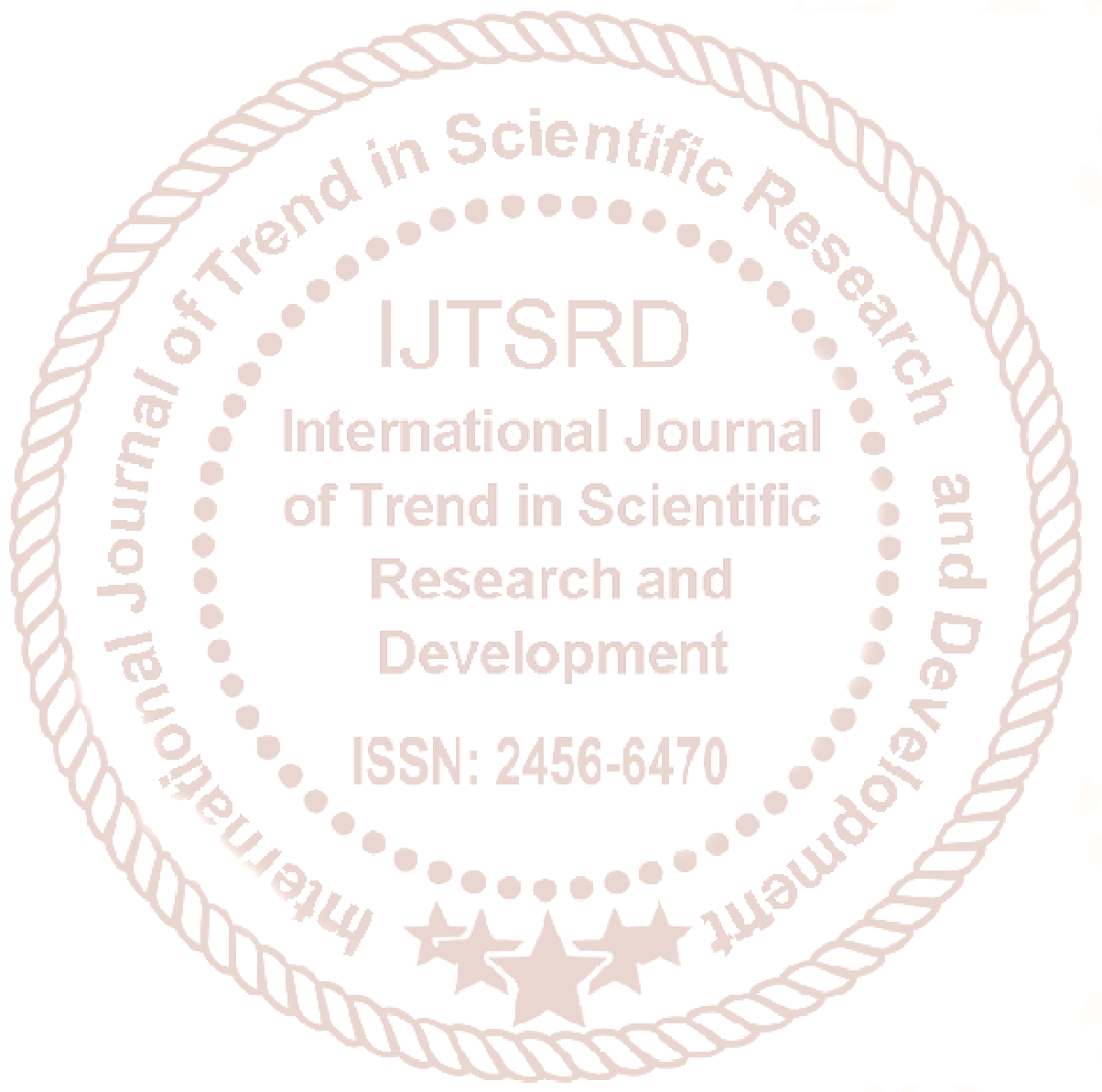

